MATEC Web of Conferences 33, 07005 (2015)

DOI: $10.1051 /$ matecconf/ 20153307005

(c) Owned by the authors, published by EDP Sciences, 2015

\title{
Austenite reconstruction via EBSD measurements: a tool to understand low Carbon martensite steel properties
}

\author{
Lieven Bracke $^{1, a}$, Laura Moli Sanchez ${ }^{1}$ and Nicolas Bernier ${ }^{2,3}$ \\ ${ }^{1}$ ArcelorMittal Global R\&D Ghent, J.F. Kennedylaan, 9060 Zelzate, Belgium \\ ${ }^{2}$ University Grenoble Alpes, 38000 Grenoble, France \\ ${ }^{3}$ CEA, LETI, MINATEC Campus, 17 rue des Martyrs, 38054 Grenoble Cedex 9, France
}

\begin{abstract}
The basic characterization of the austenite grain size and shape prior to quenching to martensite was already used in the past to optimize the mechanical properties and impact toughness of low Carbon martensitic steel. This basic characterization can typically be done by optical microscopy. To better understand the mechanisms that generate the different properties, however, a more detailed analysis is required. An algorithm has been developed to calculate the austenite orientation starting from the martensite orientation measured by EBSD. The method has been applied to explain the different properties of martensite after austenitization and quenching and of direct quenched martensite with different levels of accumulated strain. It is shown that a small austenite size is needed to improve the impact toughness, regardless of the process route. The strength is strongly depending on the dislocation density in the austenite.
\end{abstract}

\section{Introduction}

The strength and the properties of hot rolled martensitic low carbon steels are known to depend strongly on the austenite condition prior to quenching [1]. Two main processes can be used to produce martensitic steel plates [2]. The first one is through re-austenitizing and quenching (RAQ). In this case, the austenite is fully recrystallized and equiaxed before quenching. Alternatively, the quenching can also be done immediately after hot rolling, i.e. direct quenching (DQ). For the DQ processes, three main conditions can be applied. In case of a finish rolling temperature well above temperature of non-recrystallization $\left(\mathrm{T}_{\mathrm{nr}}\right)$, the quenching occurs from coarse, recrystallized austenite. In steel plate production context this, is often referred to as "hot rolling" (HR). In case the finish rolling temperature is at or just above the $\mathrm{T}_{\mathrm{n}}$, the quenching starts from a finegrained, equiaxed austenite. This process is called "recrystallization controlled rolling" (RCR). Note, however, that this process has a tendency to be unstable because of its sensitivity to small process variations [1]. For a finish rolling temperature below $\mathrm{T}_{\mathrm{n}}$, the quenching is done from deformed austenite and the process is called "controlled rolling" (CR). A schematic overview of the four processes is given in Figure 1.

Characterization and understanding the austenite condition prior to quenching is a key factor to optimize the strength and toughness of martensitic steels. Chemical etching has traditionally been applied to reveal the prior austenite grain boundaries (PAG) [3]. By using this
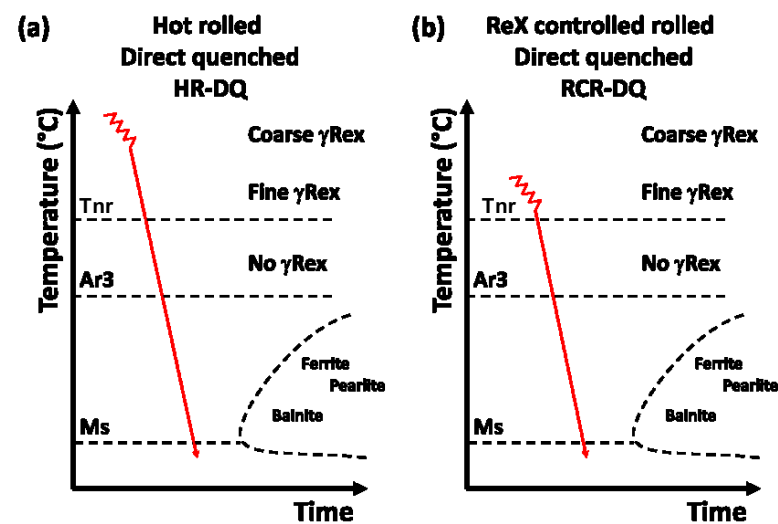

(c)

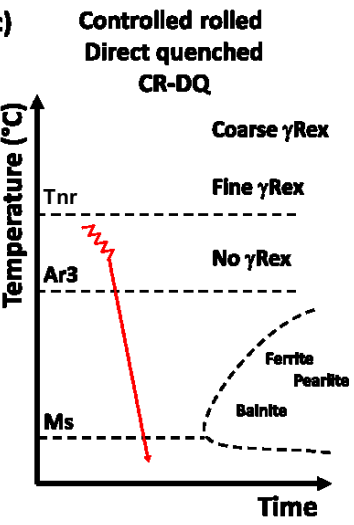

(d) Re-austenitised - quenched
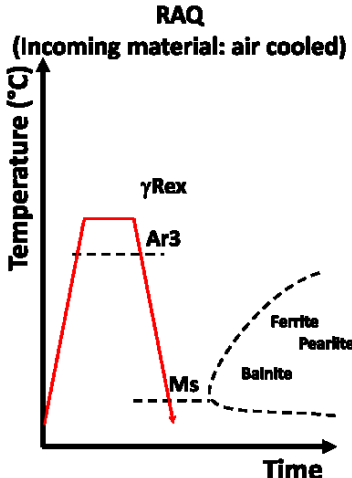

Figure 1 Schematic representation of the four types of processes that can be used to produce martensitic steel

\footnotetext{
${ }^{\mathrm{a}}$ Corresponding author: lieven.bracke@arcelormittal.com
} 
technique, valuable information on size and morphology can be obtained. More recently, however, methods for reconstructing the austenite structure have been developed based on Electron Backscattered Electron Diffraction (EBSD) maps of martensite [4-7]. The major advantage of these methods is that crystallographic information of the austenite can be retrieved and that orientation data obtained can be used to analyse and quantify microstructural features using standard EBSD software.

In this study, the four different processes for producing hot rolled low carbon martensite steel plates are compared in terms of strength and toughness. The variations in properties are explained based on the analysis of the prior austenite condition.

\section{Austenite reconstruction method}

The detailed description of the austenite reconstruction method used can be found in ref. [7]. The main principles are summarized below. The starting point for the reconstruction algorithm is an ESBD scan of a martensitic or bainitic sample. The algorithm is subdivided into three major steps. In a first step, the orientation relationship (O-R) between martensite $(\alpha)$ and the parent austenite $(\gamma)$ is determined because deviations have been observed from the traditionally used Kurdjumov-Sachs (K-S) (i.e., $\{111\} \gamma / /\{110\} \alpha$ and $<110>\gamma / /<111>\alpha)[8]$ or Nishiyama-Wassermann $(\mathrm{N}-$ W) (i.e., $\{111\} \gamma / /\{110\} \alpha$ and $<211>\gamma / /<110>\alpha$ ) [9]. The approach as proposed by Miyamoto et al. [10] has been applied. The average deviation angle for a number of experimental $\alpha$ orientations within one $\gamma$ grain is minimized with respect to the theoretically predicted $\mathrm{O}$ $\mathrm{R}$. This can be done in a number of cropped areas that can be identified as coming from one single $\gamma$ grain, based on visual appearance of grain boundaries in the EBSD scan. The expected accuracy of the O-R determination is $2^{\circ}$ or better.

The second step consists of identification of the $\gamma$ nuclei throughout the complete EBSD scan. For this, the scan is subdivided in cropped sections in which the $\gamma$ orientation is calculated for each pixel using the previously defined O-R. Within that cropped area, the two most occurring orientations are selected and compared with each other. If the most occurring orientation sufficiently outnumbers to other one, the variant is selected. As a consequence, a cropped area containing two prior austenite grains will not be attributed an orientation. Cropped areas are overlapping during this process.

In the third and final step, the nuclei identified in the previous step are expanded into full grains. To obtain this, the 24 possible austenite orientations of each measured martensite pixel are calculated using the orientation relationship defined in the first step and compared with the variant determined in the second step for the cropped area to which the pixel belongs. The variant leading to the lowest misorientation compared to the nucleus is selected. The indexed pixel gets its final austenite orientation based on its original martensite orientation and the variant as determined above. The expansion of the nucleus stops when the pixel shows a too large misorientation with the nucleus orientation.

\section{Materials and procedures}

$80 \mathrm{~kg}$ blocks were cast in a vacuum induction furnace. The chemical composition is given in Table 1. The $\mathrm{A}_{\mathrm{c} 3}$ temperature of the steel is $819^{\circ} \mathrm{C}$, as determined via dilatometry. The $\mathrm{T}_{\mathrm{nr}}$ of the steel is estimated at $930^{\circ} \mathrm{C}$. Hot rolling consisted of 6 to 8 passes. Reheating prior to hot rolling was at $1250^{\circ} \mathrm{C}$. The hot rolling from $40 \mathrm{~mm}$ to $5 \mathrm{~mm}$ was done in 6 passes.

For the direct quenched (DQ) sheets, the temperature of the last pass (FRT) was between $1000^{\circ} \mathrm{C}$ (DQ-HR), $930^{\circ} \mathrm{C}$ (DQ-RCR) or $860^{\circ} \mathrm{C}$ (DQ-CR). The cooling rate was sufficient to obtain fully martensitic microstructures in all cases and the quenching was done down to room temperature.

In case of the re-austenitized and quenched (RAQ) material, the FRT was $910^{\circ} \mathrm{C}$, followed by air cooling to room temperature. Subsequently, the sheet was austenitized at $880^{\circ} \mathrm{C}$ for $15 \mathrm{~min}$. and quenched in water down to room temperature.

Table 1 Chemical composition of the steel (all in wt $\%$, balance : $\mathrm{Fe}+$ impurities)

\begin{tabular}{|c|c|c|c|}
\hline $\mathbf{C}$ & $\mathbf{M n}$ & $\mathbf{S i}$ & $\mathbf{C r}$ \\
\hline $0.12-0.15$ & $1.3-1.7$ & $<0.3$ & $<0.50$ \\
\hline $\mathbf{C u}+\mathbf{N i}$ & $\mathbf{B}$ & $\mathbf{T i}$ & $\mathbf{N}$ \\
\hline Res. & 0.002 & 0.030 & 0.005 \\
\hline
\end{tabular}

Tensile testing was done on proportional specimens according to EN ISO 6892-1 and Charpy impact testing was done on sub-sized specimens following ISO 148-1. Test temperature was $-40^{\circ} \mathrm{C}$. Microstructural investigations were performed on polished RD-ND cross sections.

Optical microscopy after Béchet-Beaujard etching was used to reveal the prior austenite grain (PAG) structure. The prior austenite grain size (PAGS) was determined according to the linear intercept method E112. EBSD measurements were performed on a JEOL JSM-7001F FEG-SEM at $20 \mathrm{kV}$ equipped with a HKL Nordlys camera for the detection of backscattered diffraction patterns. The angular resolution of the equipment is $2^{\circ}$. The step size used for the scan was $0.2 \mu \mathrm{m}$ and the scanned area was $250 \times 200 \mu \mathrm{m}^{2}$. The measured martensite orientation maps were used to reconstruct the prior austenite grain structure following the procedure described above. 


\section{Results}

\subsection{Mechanical properties}

The tensile strength and the Charpy V impact energy at $40^{\circ} \mathrm{C}$ are shown in Figure 2 for the different processing conditions. The strength increases with a lower finish rolling temperature for the DQ materials. The RAQ 880 material has the lowest strength. The Charpy impact toughness does not vary significantly for the DQ, although the DQ 930 material shows a slightly worse performance. The RAQ 880 shows by far the best toughness result.

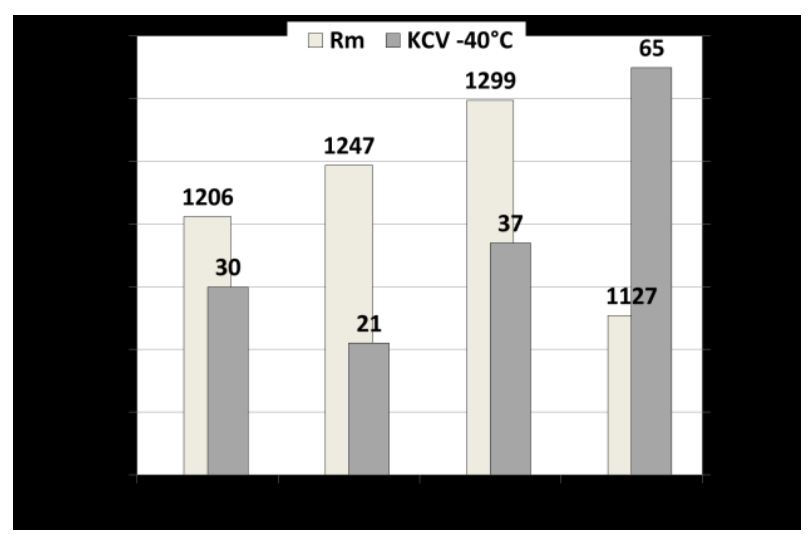

Figure 2 Tensile strength and Charpy V impact energy at $-40^{\circ} \mathrm{C}$ for the different processing conditions.

\subsection{Characterization of the prior austenite}

Figure 3 shows the EBSD maps as-measured in the martensitic conditions, whereas the reconstructed austenite maps are shown in Figure 4. For comparison, optical micrographs of the PAG are presented in Figure 5. The morphological correspondence is obvious between both methods for revealing the prior austenite structure. The average PAGS calculated from EBSD reconstructed maps is typically lower than for the PAGS via the linear intercept method on optical micrographs, as shown in Table 2. The trends however are similar. For the EBSD result, the PAGS smaller than $3.5 \mu \mathrm{m}$ have been discarded because the reconstruction routine creates a large number of artificial small grains. For the currently analyzed scans, grains below $3.5 \mu \mathrm{m}$ in equivalent diameter represent about $90 \%$ of the number of grains, whereas they only represent about $3 \%$ of the area fraction.

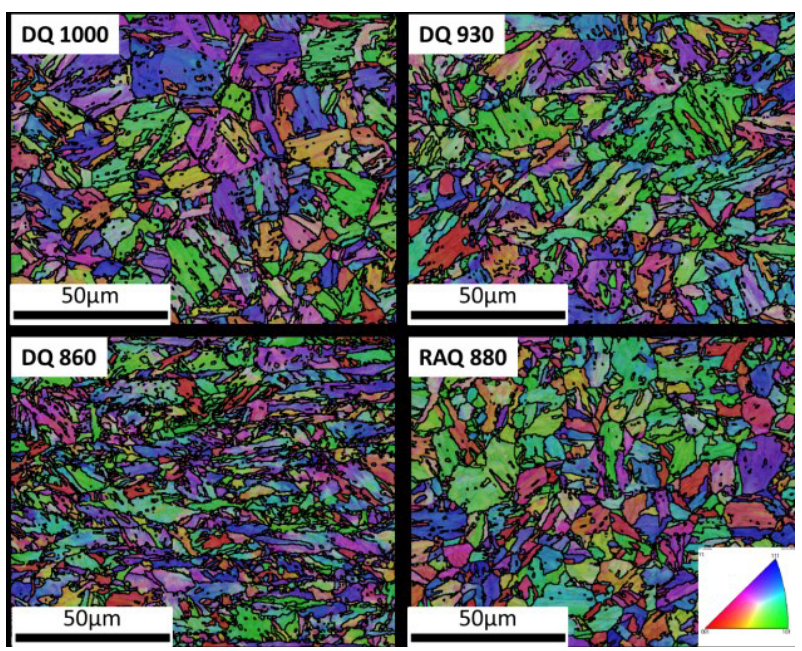

Figure 3 EBSD maps for the different processing conditions : originally measured martensite structure. (IPF colouring, black lines : $15^{\circ}$ grain boundaries)

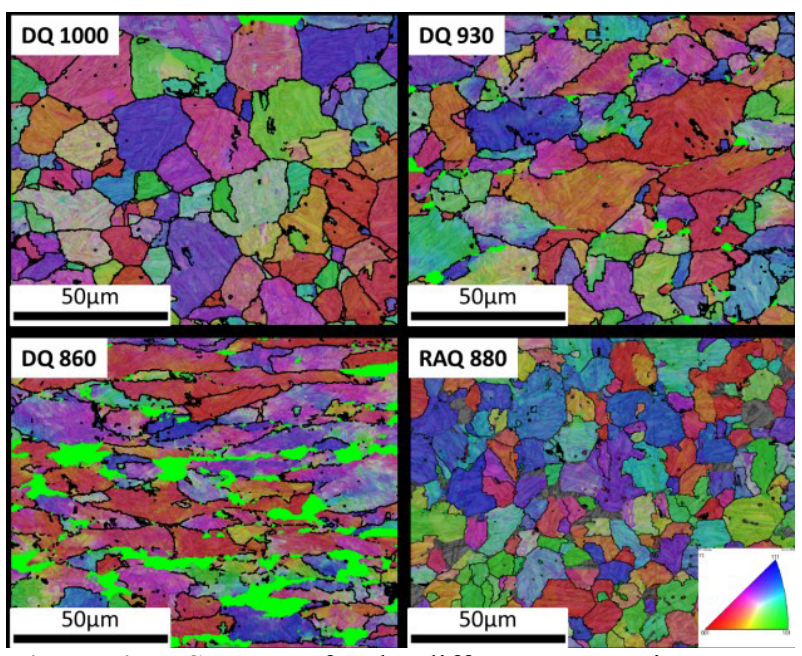

Figure 4 EBSD maps for the different processing conditions : reconstructed austenite structure. (IPF colouring, black lines : $15^{\circ}$ grain boundaries) (Bright green areas in DQ 930 and DQ 860 : unreconstructed areas)

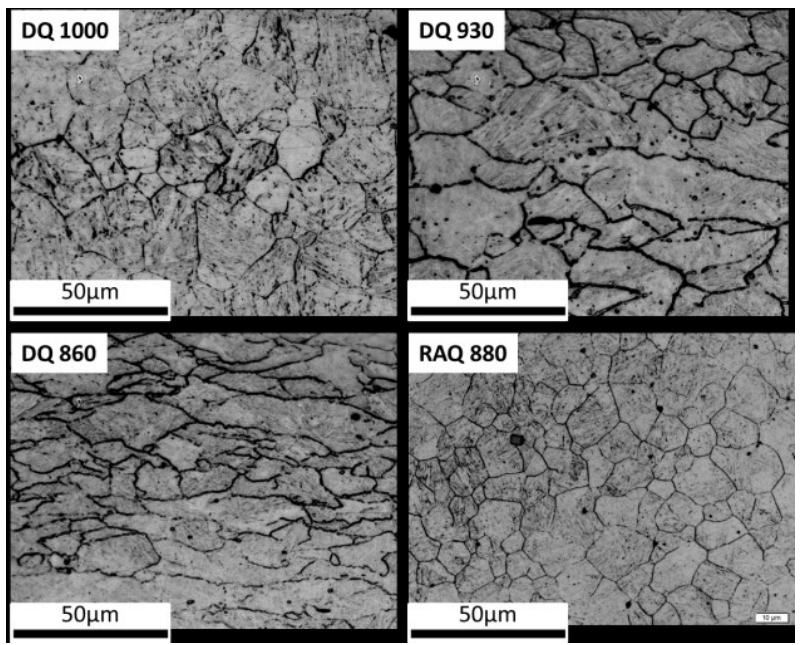

Figure 5 Optical micrographs of the prior austenite grains after Béchet-Beaujard etching for the different processing conditions (RD-ND sections) 
Table 2 Comparison between PAGS (equivalent diameter) via ASTM 112 after Béchet-Beaujard (B-B) etching and via analysis of reconstructed EBSD maps using the HKL software.

\begin{tabular}{|c|c|c|}
\hline & \multicolumn{2}{|c|}{$\begin{array}{c}\text { Average PAGS } \\
(\boldsymbol{\mu} \mathrm{m})\end{array}$} \\
\hline & B-B & EBSD \\
\hline DQ1000 & 18 & 12 \\
\hline DQ 930 & 16 & 13 \\
\hline DQ 860 & 17 & 11 \\
\hline RAQ 880 & 10 & 8 \\
\hline
\end{tabular}

The PAGS distributions in Figure 6 show that the smaller average PAGS of the RAQ880 is mainly a consequence of the relatively small variation in PAGS compared to the DQ samples. In the DQ samples, significant fractions of relatively large grains are present. The expected larger grain size of the DQ 1000 ("HR DQ") compared to the DQ 930 ("RCR DQ") was not observed. This suggests that even higher rolling temperature than $1000^{\circ} \mathrm{C}$ are required to induce significant grain growth, at least for similar rolling passes and interpass times. The absence of a size difference between the DQ 930 and DQ 860 was expected since the grains are only pancaked when rolling below $\mathrm{T}_{\mathrm{nr}}$. Grain refinement would require a recrystallization process, which is impossible by definition of $\mathrm{T}_{\mathrm{nr}}$.

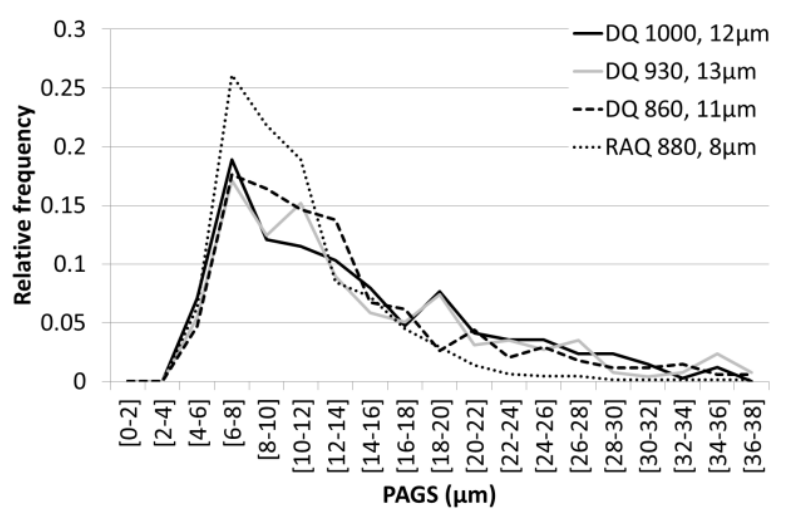

Figure 6 Prior austenite grain size distributions, based on reconstructed austenite EBSD maps.

The misorientation angle distribution determined from the measured martensite EBSD maps show a higher fraction of low angle misorientations with reducing FRT, cf. Figure 7. The RAQ880 material shows the lowest fraction of low angle misorientations. This indicates a higher dislocation density in martensite [11] with reducing FRT, which is in line with the expectation that martensite inherites the dislocations formed in austenite $[12,13]$.

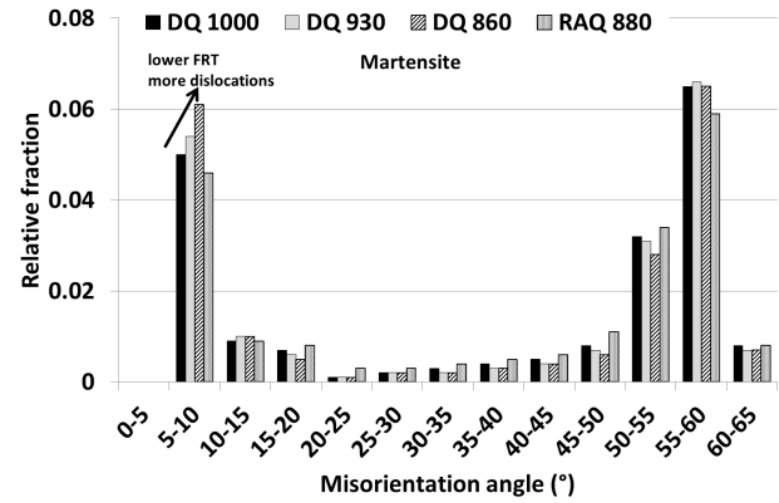

Figure 7 Misorientation angles, based on martensite EBSD map.

This higher dislocation density in austenite for the DQ 860 is visualized in Figure 8 as higher misorientation within a PAG compared to the RAQ 880 condition. Note that with expected accuracy of the O-R determination $\left(<2^{\circ}\right)$ and the measurement $\left(2^{\circ}\right)$, the differences observed can be considered as relevant. The effect can also be shown more quantitatively by the distribution of the misorientation within a PAG compared to the average orientation of that PAG, as shown in Figure 9. The DQ 1000 and the RAQ 880 are expected to have the lowest dislocation density, which corresponds to a low misorientation within most of the grains. The DQ 860 has the highest dislocation density, which translates into a large fraction of grains with a high misorientation within the PAG.
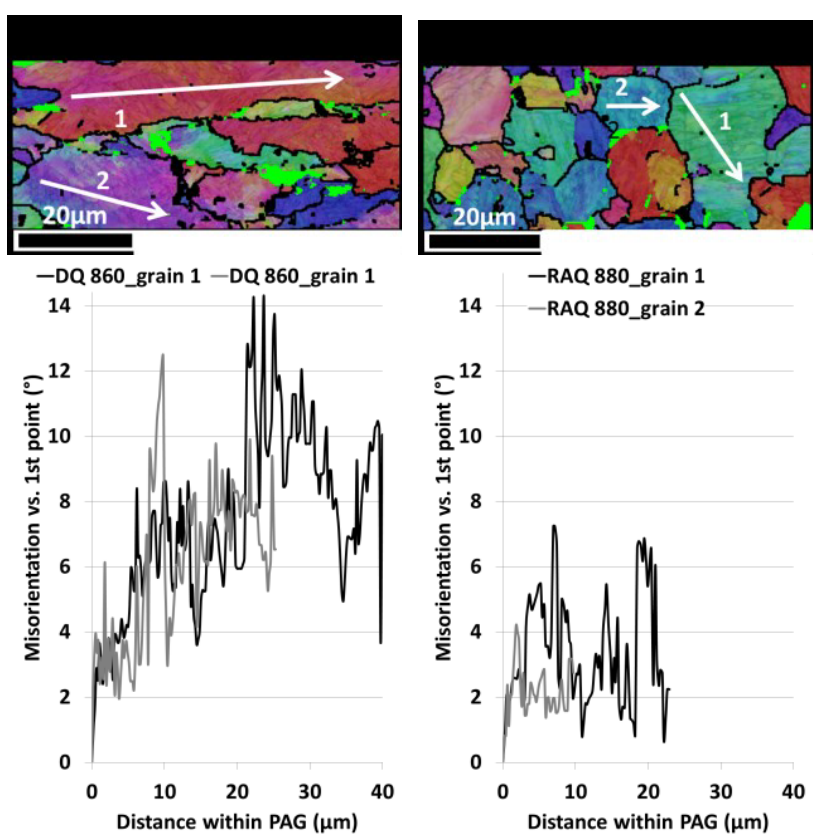

Figure 8 Details of reconstructed austenite maps. Misorientation within PAG for (left) two grains in DQ 860, (right) RAQ 880. 


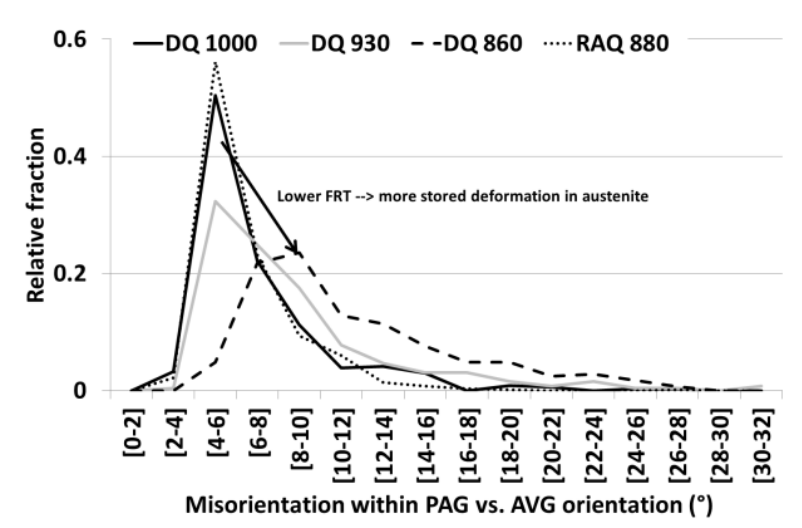

Figure 9 Misorientation within PAG compared with average orientation in each grain.

\section{Discussion}

The strength and toughness differences between the four different processing conditions can be explained through analysis of the prior austenite condition. Note that a detailed analysis of the martensite substructure - which can be considered as a direct consequence of the prior austenite condition - has not been performed. Furthermore, the crystallographic textures were not very pronounced and are not taken into account either.

The strength of the DQ materials increases with decreasing FRT whereas the lowest strength is found for the RAQ 880 material. This is in line with expectations from the literature, e.g. [2, 11]. The PAGS is similar for all the DQ materials and larger than for the RAQ 880, ruling out the PAGS as such as the main factor controlling the strength of martensite in these materials (Figure 10). Based on the martensite misorientation angles (Figure 7) and the orientation variations in the reconstructed austenite grains (Figure 8 and Figure 9), it has been shown that that the dislocation density increases with decreasing FRT and the RAQ 880 has a lower dislocation density than the DQ materials. Figure 11 indicates that it is indeed the dislocation density that is the major influence on the strength of the martensite.

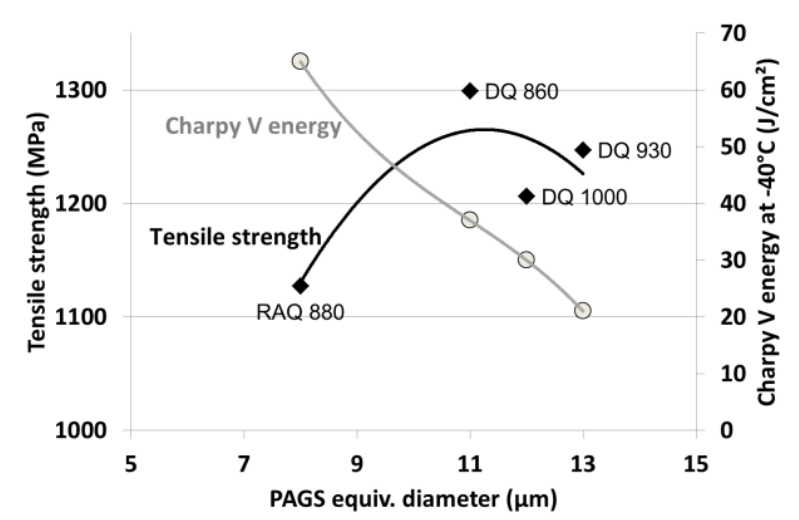

Figure 10 Tensile strength and Charpy V impact toughness vs. PAGS equivalent diameter (determined via reconstructed austenite map).

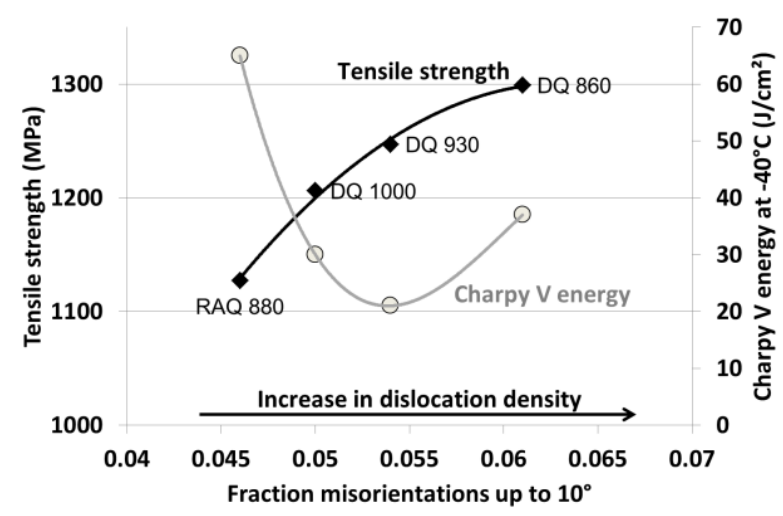

Figure 11 Tensile strength and Charpy V impact toughness vs. low angle misorientations in martensite.

The Charpy V impact toughness was low for all the DQ materials, whereas the RAQ 880 performed significantly better. No correlation with the dislocation density is apparent, as can be seen from Figure 11, but a good correlation is found with the average PAGS equivalent diameter in Figure 10.

\section{Conclusions}

Four different processes have been applied to the same steel to generate a martensitic microstructure starting from different prior austenite conditions. These different processes lead to different strength and toughness of the martensite.

An in-house developed routine has been applied to reconstruct the prior austenite structure from EBSD maps measured in the martensite condition.

It was shown that the strength differences between the martensitic materials are mainly determined by the dislocations inherited from the austenite. The toughness is mainly driven by the size of the prior austenite grains.

\section{References}

1. L. Bracke, W. Xu, T. Waterschoot, Mat. Today: Proceedings Icomat 2014, to be published

2. R. K. Weiss, S. W. Thompson, in K.A. Taylor, S.W. Thompson, F.B. Fletcher (Eds.), Physical Metallurgy of Direct Quenched Steels, TMS, Warrendale, (1993), 107-138

3. S. Béchet, L. Beaujard, Rev. Met. 52 (1955), 830836

4. C. Cayron, B. Artaud, L. Briottet, Mater. Charact. 57 (2006), 386-401

5. G. Miyamoto, N. Iwata, N. Takayama, T. Furuhara, Acta Mater. 58 (2010), 6393-6403

6. L. Germain, N. Gey, R. Mercier, P. Blaineau, M. Humbert, Acta Mater. 60 (2012), 4551-4562

7. N. Bernier, L. Bracke, L. Malet, S. Godet, Mater. Charact. 89 (2014), 23-32

8. G. Kurdjumov, G. Sachs, Z. Phys. 64 (1930), 325343

9. Z. Nishiyama, Sci. Rep. 23 (1934), 636-649 
10. G. Miyamoto, N. Takayama, T. Furuhara, Scr. Mater. 60 (2009), 1113-1116

11. Y. Zhao, J. Shi, W. Cao, M. Wang, G. Xie, J. Zheijang Univ. Sci. A 11 (2010), 776-781

12. A. S. Schulz-Beenken, J. Phys. IV C5 (1997), 359366

13. T. Maki, in K.A. Taylor, S.W. Thompson, F.B. Fletcher (Eds.), Physical Metallurgy of DirectQuenched Steels, TMS, Warrendale, (1993), 3-16 